\title{
Observations of recurrent cosmic ray decreases during solar cycles 22 and 23
}

\author{
P. Dunzlaff, B. Heber, A. Kopp, O. Rother, R. Müller-Mellin, A. Klassen, R. Gómez-Herrero, and \\ R. Wimmer-Schweingruber \\ Institut für Experimentelle und Angewandte Physik, Christian-Albrechts-Universität zu Kiel, 24118 Kiel, Germany \\ Received: 28 November 2007 - Revised: 1 September 2008 - Accepted: 1 September 2008 - Published: 15 October 2008
}

\begin{abstract}
During solar cycle 22, the modulation of several hundred $\mathrm{MeV}$ galactic cosmic rays (GCRs) by recurrent and transient cosmic ray decreases was observed by the Ulysses spacecraft on its descent towards the solar south pole. In solar cycle 23 , Ulysses repeated this trajectory segment during a similar phase of the solar cycle, but with opposite heliospheric magnetic field polarity. Since cosmic ray propagation in the heliosphere should depend on drift effects, we determine in this study the latitudinal distribution of the amplitude of recurrent cosmic ray decreases in solar cycles 22 and 23. As long as we measure the recurrent plasma structures in situ, we find that these decreases behave nearly the same in both cycles. Measurements in the fast solar wind, however, show differences: in cycle $22(A>0)$ the recurrent cosmic ray decreases show a clear maximum near $25^{\circ}$ and are still present beyond $40^{\circ}$, whereas we see in cycle $23(A<0)$ neither such a pronounced maximum nor significant decreases above $40^{\circ}$. In other words: the periodicity in the cosmic ray intensity, which can be clearly seen in the slow solar wind, appears to vanish there. Theoretical models for drift effects, however, predict quite the opposite behaviour for the two solar cycles. To closer investigate this apparent contradiction, we first put the visual inspection of the data onto a more solid basis by performing a detailed Lomb (spectral) analysis. The next step consists of an analysis of the resulting periodicities at $1 \mathrm{AU}$ in order to distinguish between spatial and temporal variations, so that we can obtain statements about the question in how far there is a correlation between the in-situ data at $1 \mathrm{AU}$ and those measured by Ulysses at larger latitudes. We find a good correlation being present during cycle 22 , but not for cycle 23. As one potential explanation for this behaviour, we suggest the difference in the coronal hole structures between the cycles 22 and 23 due to a large, stable coronal hole structure, which is present during cycle 22 ,
\end{abstract}

Correspondence to: P. Dunzlaff

(dunzlaff@physik.uni-kiel.de) but not in cycle 23. We support this possibility by comparing Yohkoh SXT and SOHO EIT maps.

Keywords. Interplanetary physics (Cosmic rays)

\section{Introduction}

During a magnetic storm Forbush (1937) discovered that the cosmic ray intensity measured simultaneously at two stations went down by several percent and showed a characteristic profile on a time scale of 2-3 days. These short term decreases in the GCR flux were first thought to be of Terrestrial origin, but the observation of a second type of decreases, recurring with a period of 27 days (cf. Simpson, 1954), also suggested an influence of the solar dipole field. In his detailed analysis Simpson (1954), however, could show that both types of cosmic ray decreases are caused by processes in the interplanetary medium: the first type of decreases is stronger, show a more irregular structure and occur more or less as singular events as mentioned above. These so-called transient or Forbush decreases are caused by interplanetary coronal mass ejections (ICMEs). The cosmic ray decreases of the second type have their origin in corotating interaction regions (CIRs), which are generated by a fast solar wind ramming into a slower flowing one ahead, leading to a structure being stable for several solar rotations. Cosmic ray decreases that are caused by CIRs can hence repeatedly be observed in space, so that they appear as groups with a periodicity of 27 days (see for example Heber et al., 1999; Richardson, 2004, and references therein). They are, thus, referred to as recurrent decreases, reflecting also their more regular, almost periodic structure

In this study, we concentrate on recurrent cosmic ray decreases and their periodic nature. On the one hand, they have been observed in situ near $1 \mathrm{AU}$ in the ecliptic plane by spacecraft like ACE or SOHO (cf. Kunow et al., 1995;

Published by Copernicus Publications on behalf of the European Geosciences Union. 

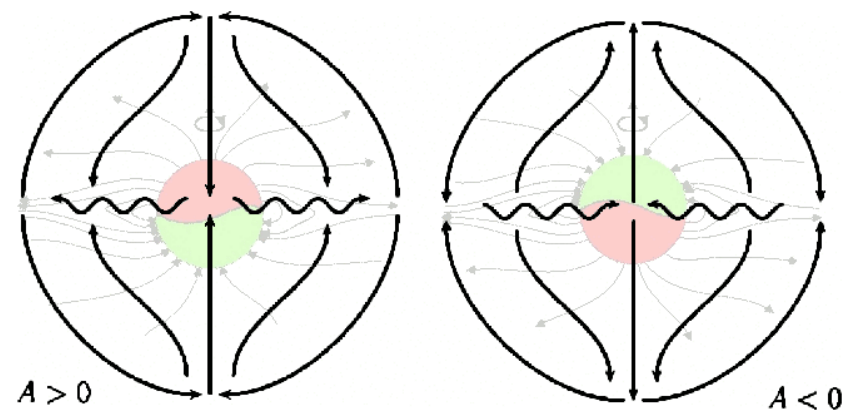

Fig. 1. The drift motion of positively charged cosmic ray particles in the heliosphere for solar cycles with $A>0$ (left panel) and $A<0$ (right panel). The Sun (not to scale), the solar magnetic field, and its polarity are indicated in the background image.

Richardson et al., 1999). On the other hand the out-of-plane orbit of Ulysses offers the opportunity to observe such structures not only farther away from the Sun, but also at higher latitudes. As the measurement of cosmic ray decreases at higher latitudes, however, can happen only remotely (e.g. Fisk and Jokipii, 1999), because the modulating structure is no longer present locally, the question of the transport of charged particles within the heliosphere becomes important.

In order to address this question, we can also make use of results for Forbush decreases as far as only the propagation of these perturbations through the interplanetary medium is concerned: Le Roux and Potgieter (1991) used a timedependent particle-transport code in order to simulate Forbush decreases. Their finding that amplitude and recovery phase depend on the polarity of the heliospheric magnetic field point out the importance of drift effects. During an $A>0$ solar magnetic epoch, i.e. when the solar magnetic field is pointing out over the solar north pole (cf. Fig. 1), drift models predict that positively charged particles drift predominantly inward through the solar polar regions and then outward through the equatorial regions along the heliospheric current sheet (Jokipii et al., 1977). In an $A<0$ solar magnetic epoch, these particles drift mainly into the inner heliosphere along the heliospheric current sheet and then outward through the polar regions (Potgieter and Moraal, 1985) as sketched in Fig. 1.

The first opportunity to verify the model of Le Roux and Potgieter (1991) by observing the cosmic ray modulation at higher latitudes was the initial descent of the Ulysses spacecraft in 1992 to 1994 from the equatorial plane towards the solar south pole, i.e. during the $A>0$ solar cycle 22. A welldefined temporal intensity variation of cosmic rays in connection with CIRs was observed (Kunow et al., 1995). According to the drift motion shown in the left panel of Fig. 1, this variation should decease at higher latitudes. Moreover, CIRs are limited to latitudes where the slow solar wind has been observed (in the range $30^{\circ}-40^{\circ}$ (Paizis et al., 1999) or perhaps smaller, cf. Phillips et al., 1995). Surprisingly, however, Ulysses still observed a periodic modulation with a roughly 26-day recurrence even beyond these latitudes.

In order to solve this puzzle as well as in consideration of the observed small latitudinal gradients, Jokipii and Kóta (1995) and Fisk (1996) proposed a large perpendicular particle transport by diffusion or magnetic connection to take place. Thus, longitudinal intensity variations can actually be transported to high latitudes.

According to cosmic ray transport models (Alania et al., 2005; Gil et al., 2005), the amplitudes of the recurrent decreases are expected to be larger for cycles with $A<0$ than for those with $A>0$. In contrast to this expectation the comparison of galactic cosmic ray data close to $1 \mathrm{AU}$ for different solar cycles (Richardson et al., 1999; Alania et al., 2005; Richardson, 2004), however, showed quite the opposite: the amplitudes observed during the $A<0$ cycles 21 and 23 turned out to be smaller than those observed during the $A>0 \mathrm{cy}$ cles 20 and 22 .

Since Ulysses is the only spacecraft, which has measured the GCR decreases both in an $A>0$ and an $A<0$ magnetic epoch in situ as well as remotely, it will add important informations on these contradictions. Furthermore, the amplitude provides also informations about the spatial distribution of the cosmic rays: Paizis et al. (1999) and Zhang (1997) found a close correlation of the amplitude with the radial and latitudinal gradients, where it is important to note that they found a maximum intensity at latitudes around $25^{\circ}-30^{\circ}$.

Based on the new Ulysses results for solar cycle 23, our study is arranged in the following way: in Sect. 2 we briefly describe the trajectory and the instruments on board of Ulysses, followed by a report of the data in Sect. 3. Section 4 is devoted to the analysis of the data and their possible conclusions: Sect. 4.1 presents a comparison of the amplitudes for both solar cycles and their latitudinal dependencies. A detailed mathematical analysis of the observed periodicities is given in Sect. 4.2. In order to properly classify our observations, we must separate temporal from spatial variations. This is done in Sect. 4.3 by comparing the Ulysses data with those obtained with SOHO and ACE at $1 \mathrm{AU}$, keeping in mind that CIRs (as the main cause for recurrent decreases) do not become fully developed until $1 \mathrm{AU}$, so that the Ulysses measurements between 2 and $5 \mathrm{AU}$ are crucial to investigate local CIR effects, whereas the SOHO and ACE data provide the heliospheric background conditions. A possible explanation is suggested in Sect. 5, where perpendicular diffusion of the particles within coronal hole structures is taken into account. Finally, we summarise and discuss our findings in Sect. 6 .

\section{Ulysses - trajectory and instrumentation}

Ulysses was launched on 6 October 1990 and followed an in-ecliptic path towards Jupiter in order to be deflected in February 1992 by the gravitational field of the planet to its 
Table 1. Definition of time periods used in this study.

\begin{tabular}{ccc}
\hline & P1 & P2 \\
\hline C22 & $1992.60-1993.50$ & $1993.80-1994.60$ \\
C23 & $2004.80-2006.00$ & $2006.15-2007.00$ \\
\hline
\end{tabular}

final out-of-ecliptic orbit with a period of 6.2 years. The spacecraft reached its maximum latitude of $-80.2^{\circ}$ in mid1994 during the declining phase of solar cycle 22. The two upper panels in Fig. 2 display the radial distance and heliographic latitude of Ulysses. The shaded areas C22 and C23 indicate the two time intervals of almost identical trajectories analysed in this study, referring to the solar cycles $22(A>0)$ and $23(A<0)$. Both intervals are further divided into a period $\mathrm{P} 1$, when Ulysses was sampling both the slow and fast solar wind and a period P2, where Ulysses was exposed to only the fast solar wind ${ }^{1}$. In the following we refer, thus, to the four periods of time displayed in Table 1 .

The third panel displays the main difference between the two intervals, the oppositely directed solar polar magnetic fields. It shows the field strength in the southern as well as in the northern polar regions, taken from http://quake.stanford. edu/ $\sim$ wso/. In addition to the sign, the data also show a lower absolute value of the magnetic field strength during C23 than during C22. The fourth panel is dealing with the solar activity and shows again more similarities than differences: while the sunspot number (black line) is lower in C23 than in $\mathrm{C} 22$, the tilt angle of the solar magnetic field (red line) was somewhat higher in the second epoch, so that we may conclude that the two periods, C22 and C23, are characterised not only by almost identical trajectory segments, but also by nearly the same heliospheric conditions. With the polarity of the solar magnetic field being the only real differerence, the data analysis of these two intervals offer an almost ideal opportunity to study drift effects isolated from other influences. The Ulysses data used in this study were obtained with the Kiel Electron Telescope (KET), which is part of the Cosmic Ray and Solar Particle Investigation (COSPIN) (Simpson et al., 1992), the Solar Wind Observations Over the Poles of the Sun (SWOOPS) (Bame et al., 1992) and the Vector Helium Magnetometer (VHM) (Balogh et al., 1992) on board Ulysses. As mentioned in the introduction, we compare the Ulysses data with those obtained close to Earth. The data used for this analysis were obtained from the Solar Wind Electron, Proton \& Alpha Monitor (SWEPAM) (McComas et al., 1998), the Magnetic Field Experiment (MAG, Smith et al., 1998) on board the ACE spacecraft (Stone et al., 1998), the Electron Proton Helium Instrument (EPHIN) (Müller-

\footnotetext{
${ }^{1}$ In order to avoid ambiguities we will call P1 and P2 in the following simply the periods of the "slow" (i.e. slow and fast) and "fast" (i.e. only fast) solar wind, respectively, keeping in mind that this is strictly speaking an incorrect simplification.
}

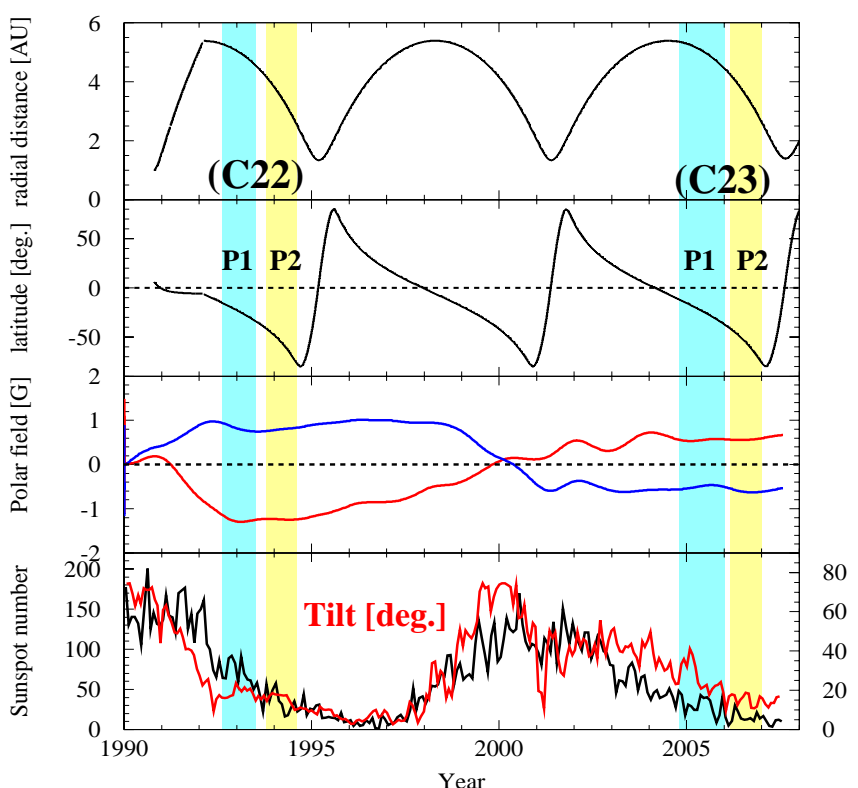

Fig. 2. From top to bottom: radial distance (in AU, first panel) and heliographic latitude (second panel) of the Ulysses orbit, solar magnetic field strength of the southern (red line) and northern (blue line)

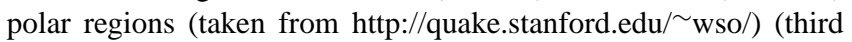
panel), as well as sunspot number (black, left hand axis) and the tilt angle (red, right hand axis) of the solar magnetic field (fourth panel). Marked by colour shading are the two periods C22 $(A>0)$ and C23 $(A<0)$ compared in this study, when Ulysses was at high southern heliographic latitudes during the solar cycles 22 and 23, respectively. The two subsections P1 (light blue) and P2 (yellowgreen) within both time intervals indicate whether Ulysses was exposed to the slow (P1) and fast (P2) solar wind, respectively.

Mellin et al., 1995) on board SOHO, and the ground based Kiel and Moscow Neutron Monitors (Clem and Dorman, 2000).

\section{Observations}

An overview of the Ulysses measurements made in C22 and C23 is displayed in the left and right panels of Fig. 3, respectively. Both plots show, from top to bottom, the solar wind speed (SWOOPS), the magnetic field strength (VHM), 250$2000 \mathrm{MeV}$ protons (with the smoothed count rate plotted in red) and the detrended count rate of the cosmic ray protons, $\Delta C / C$ (given in \%). In addition, the radial distance and latitude of Ulysses are displayed on top of the plots. The dashed vertical lines mark periods of 26 days (C22, left) and 24.5 days (C23, right). From these observations, it becomes evident that the solar wind speed, the magnetic field strength and the GCRs vary on time-scales close to the solar rotation period in the time intervals $\mathrm{C} 22 / \mathrm{P} 1$ and $\mathrm{C} 23 / \mathrm{P} 1$, caused by CIRs. When the spacecraft enters the region of the fast solar wind (i.e. the sub-periods P2 in our nomenclature) at 

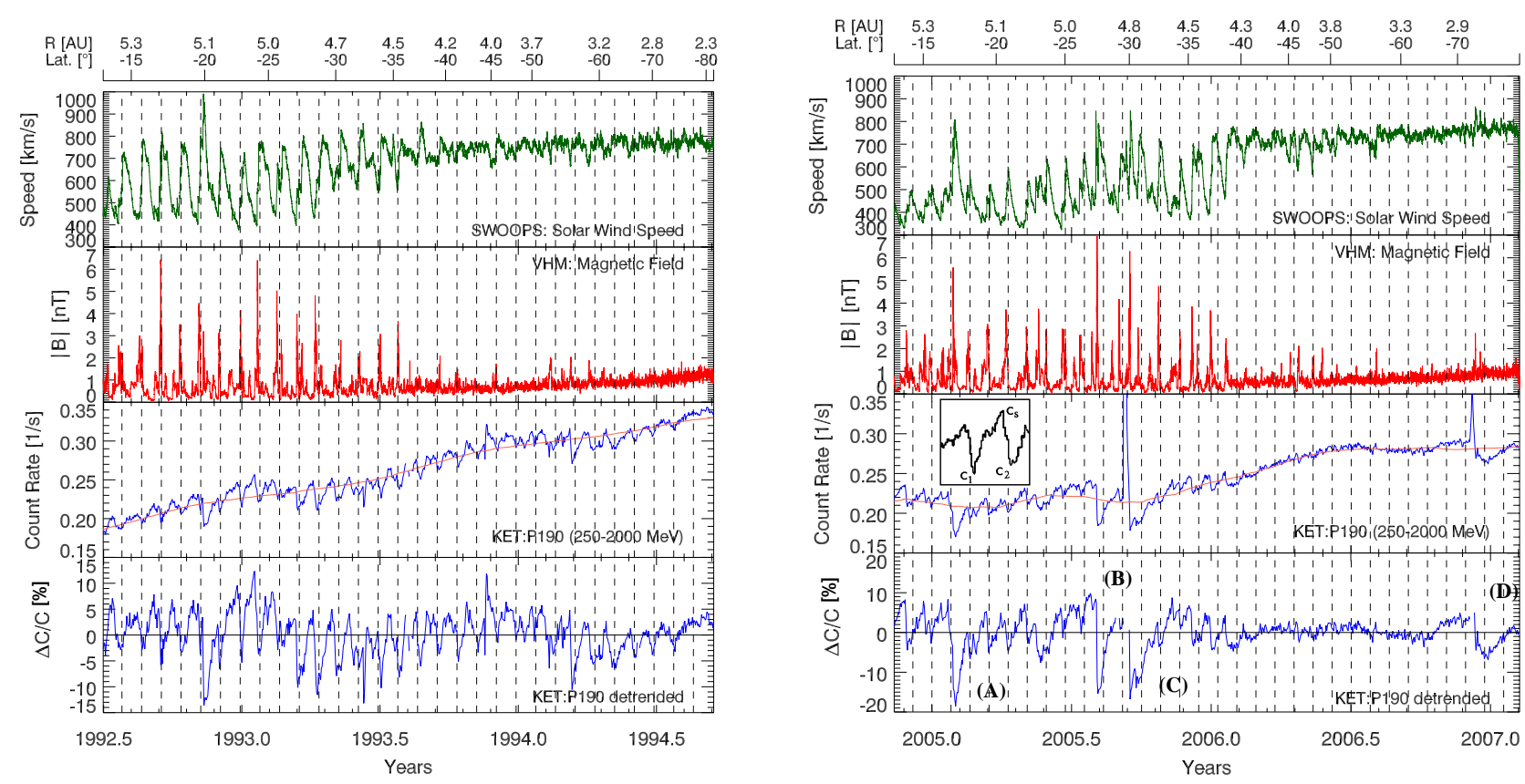

Fig. 3. Ulysses measurements during the intervals C22 (left panel) and C23 (right panel). The panels display from top to bottom hourly averages of the solar wind speed (green), the magnetic field strength (red), daily (blue) and 26-day-running mean-averaged (red) count rates of 250-2000 MeV protons as well as the long-term detrended galactic cosmic ray variation at Ulysses (blue). The labels on top of both panels give the radial distance and latitude of Ulysses' orbit. The dashed vertical lines indicate periodicities of 26 days for C22 and 24.5 days for $\mathrm{C} 23$ with the second value resulting from the Lomb analysis shown below. The letters (A) to (D) indicate four prominent transient decreases in $\mathrm{C} 23$, whereas only one prominent event (at about $20^{\circ}$ ) can be seen in $\mathrm{C} 22$. The insert in the right panel illustrates the procedure to determine the amplitudes of the GCR decreases, cf. Sect. 4.1.

about $\lesssim 40^{\circ} \mathrm{S}$, the variation seems to vanish both in the solar wind speed and the magnetic field strength in both solar cycles. The cosmic ray intensity, however, continues to be modulated in C22/P2 (cf. Kunow et al., 1995), whereas it almost vanishes in C23/P2. Lario and Roelof (2007) analysed Ulysses HISCALE data (Lanzerotti et al., 1992) and found recurrent energetic particle events for both cycles at all latitudes. While the observations in $\mathrm{C} 22$ are in good agreement with our results, they do not correspond for $\mathrm{C} 23$.

With respect to drift-dominated transport models like Jokipii et al. (1977), our new observation is, on the one hand, consistent with the theory insofar that different polarities of the solar magnetic field should also lead to different features in the GCR decreases, but on the other hand, the second observation is as surprising as already the first one was, i.e. we should have seen quite the opposite in both cases.

\section{Analysis of the data}

The Ulysses observations both for C22/P2 and C23/P2 seem to be in apparent contradiction with drift-dominated propagation models. Before studying the periodicities by using a detailed mathematical (Lomb) analysis, we will first investigate the amplitudes of the GCR decreases. The purpose is twofold: we can investigate in how far these are consistent with the theory and we can discriminate transient decreases from recurrent ones to eliminate the transient decreases from our analysis of the periodicities.

\subsection{Amplitudes}

During C23 Ulysses observed more transient and fewer recurrent cosmic ray decreases than during $\mathrm{C} 22$, as the third and fourth panels of Fig. 3 show, indicating that the solar activity in 2004 was larger than that in 1992. From the sunspot number, displayed in Fig. 2, we would expect the opposite, so that the essential role is obviously played here by the tilt angle, which was larger during $\mathrm{C} 23$ than during C22. Four outstanding transient events are marked by (A) to (D) in the right panels of in Fig. 3. They are correlated with periods of larger solar activity in January, August, September 2005, and December 2006 (Struminsky, 2007; Malandraki et al., 2007) and several ICMEs and solar energetic particle (SEP) events Lario and Roelof (2007) that have been observed in particular up to 2005.6, i.e. during C23/P1. The prominent event occuring close to $20^{\circ}$ in C22 may also be related to a SEP event Lario and Roelof (2007). 


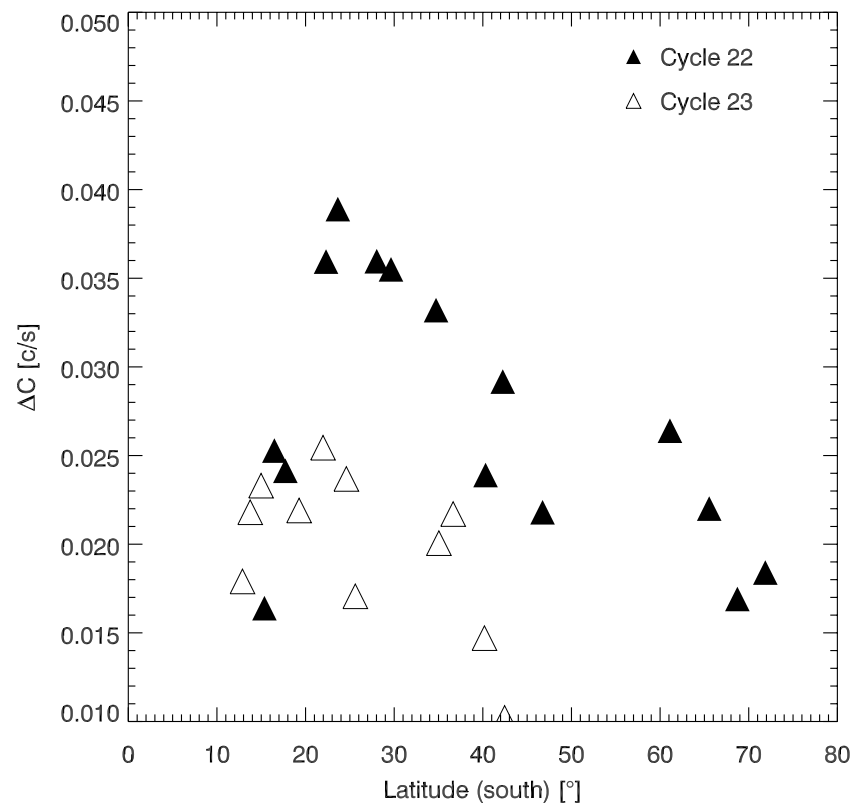

Fig. 4. Evolution of the amplitude, $\Delta c$, of recurrent GCR decreases for daily averaged 250-2000 MeV COSPIN/KET protons. The filled and open triangles represent the variations during the time intervals $\mathrm{C} 22 / \mathrm{P} 1+\mathrm{P} 2$ and $\mathrm{C} 23 / \mathrm{P} 1+\mathrm{P} 2$, respectively.

In order to determine the amplitudes of the recurrent GCR decreases we adapt the procedure suggested by Paizis et al. (1999) as illustrated in the small insert of Fig. 3: we first determine three values: the counting rate, $c_{s}$, at the positive peak in the centre of the decrease and the two negative peaks, $c_{1}$ and $c_{2}$, preceding and succeeding the positive peak, respectively. The amplitude, $\Delta c$, is then defined as the difference between $c_{s}$ and the mean value of $c_{1}$ and $c_{2}$, i.e. $\Delta c=c_{s}-\left(c_{1}+c_{2}\right) / 2$. Figure 4 shows $\Delta c$ as a function of Ulysses latitude for the complete time intervals C22 and $\mathrm{C} 23$, indicated by filled and open triangles, respectively. For $\mathrm{C} 22$, we find a good agreement with the values determined by Paizis et al. (1999). In particular, our analysis confirms their latitude dependence, with a clear maximum around $25^{\circ}$. The results for $\mathrm{C} 23$ show smaller count rates due to the enhanced solar activity and a less pronounced maximum, if any. Both data sets appear to be somewhat distorted around the transition from the slow (P1) to the fast (P2) solar wind at about $35^{\circ}-40^{\circ}$.

While the absolute values, $\Delta c$, of the amplitudes in Fig. 4, are considerably lower in $\mathrm{C} 23$ than in $\mathrm{C} 22$, the relative values, $\Delta c / c$ in the slow solar wind (periods $\mathrm{P} 1$ of each cycle), are comparable, as Fig. 5 shows. Here, the relative amplitude is plotted as a function of the reduced solar wind speed. The latter has been calculated by subtracting $380 \mathrm{~km} / \mathrm{s}(\mathrm{C} 22)$ and $300 \mathrm{~km} / \mathrm{s}$ (C23) from the measured values. In both cycles a negative slope can be seen with the gradient being somewhat stronger for reduced solar wind speeds below $200 \mathrm{~km} / \mathrm{s}$.

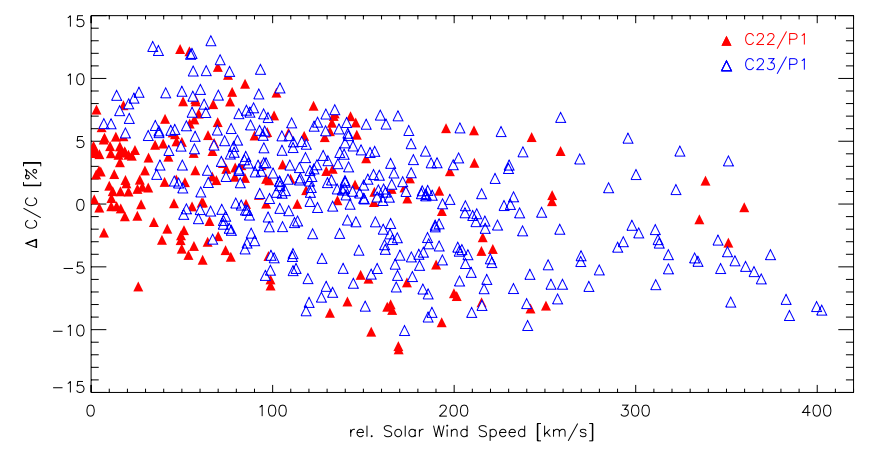

Fig. 5. Daily averages of the relative galactic cosmic ray variation as a function of the reduced solar wind speed for cycles C22/P1 (red) and C23/P1 (blue).

From this observation we may conclude the following: during C23, the GCRs were exposed to a more active Sun than during $\mathrm{C} 22$, causing the amplitudes of the GCR recurrent decreases to be smaller (Fig. 4). Eliminating these effects by considering the relative amplitude and the reduced solar wind speed, the data for the slow solar wind (P1) do not show significant differences between both cycles, so that the influence of the CIRs on the GCR modulation is (almost) the same at the position of Ulysses as long as the CIRs can be measured in situ.

\subsection{Periodicities}

In the fast solar wind (P2), however, we see much larger differences between $\mathrm{C} 22$ and $\mathrm{C} 23$ with the most remarkable one being the fact that the periodicity in the GCR modulation appears to vanish in $\mathrm{C} 23 / \mathrm{P} 2$. In order not to be limited to a simple visual inspection and a manual determination of local minima and maxima and their periodicities, we set the determination of the latter onto a solid mathematical base by applying the Lomb algorithm for being able to search also for smaller amplitude variations (Lomb, 1976).

Figure 6 shows the Lomb periodograms for the Ulysses measurements in the interval C22. The first panel shows the results for the solar wind velocity (green), the second one those for magnetic field (red), and the third one the cosmic ray flux (blue). In each panel the values for the periods P1 (slow solar wind) and P2 (fast solar wind) are displayed with dark and light colours, respectively. The results for $\mathrm{C} 23$ are shown in the same way in Fig. 7. The 26-day sidereal period of the Sun is highlighted by the vertical line, whereas the horizontal line marks a significance of $\sim 99 \%$. In order to avoid a over-plotting of two close lines, this level, which is always very close to a value of 10 , has been set to 10 in the plots. Note that the transient events (A)-(D) have been removed from the periodicity analysis for $\mathrm{C} 23 / \mathrm{P} 2$.

The periodograms for both cycles confirm the visual impression of the data displayed in Fig. 3: all three quantities show during cycle $\mathrm{C} 22$ a significant periodicity of 26 days as 


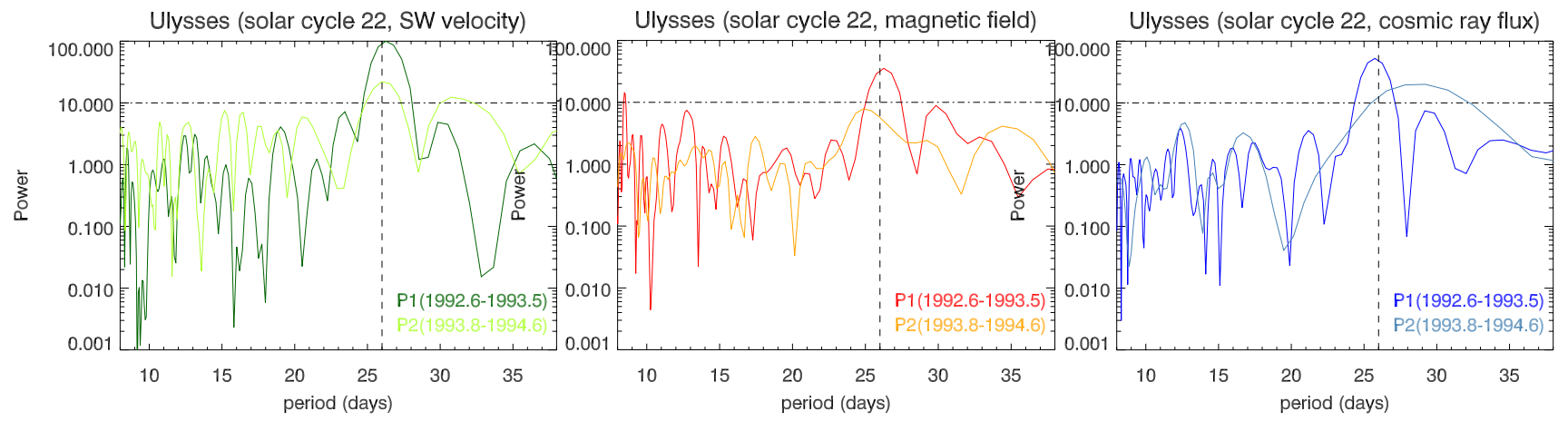

Fig. 6. Lomb periodograms for Ulysses measurements of the solar wind speed (green), the magnetic field strength (red) and the detrended galactic cosmic ray flux (blue) for the time interval C22. The light colours indicate the values for measurements in the slow solar wind (P1), light colours those in the fast solar wind (P2). The power, i.e. the spectral function normalised to the interval [0,100] (also called the Lomb values), is plotted as a function of the periodicity in days. The vertical dotted line marks a period of 26 days, the horizontal line corresponds to a significance level of $99 \%$.

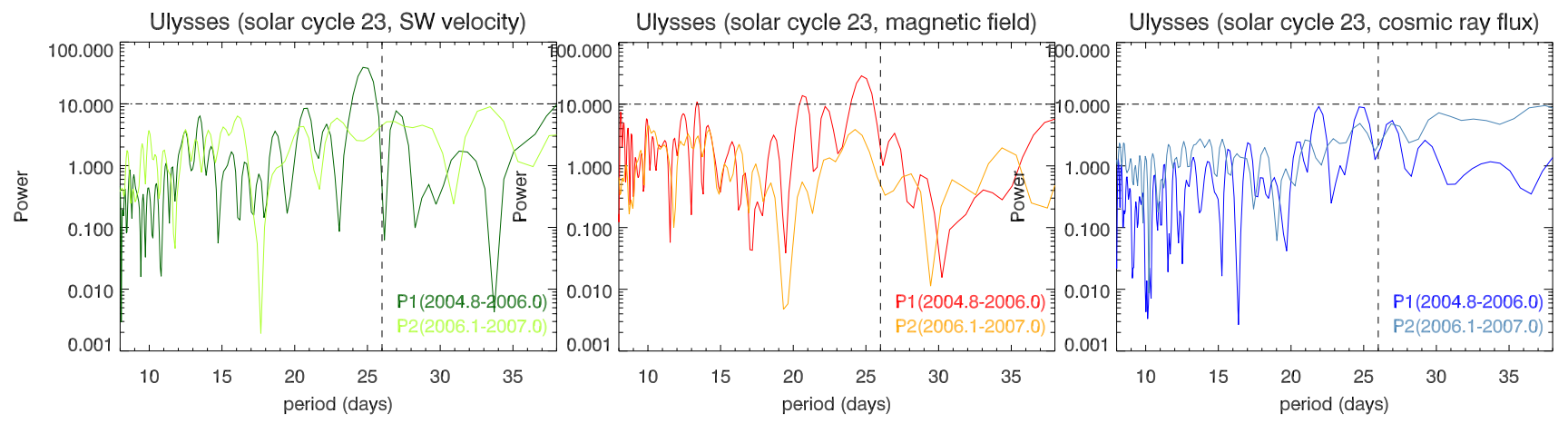

Fig. 7. Lomb periodograms for Ulysses measurements in the time interval C23 in the same representation as in Fig. 6.

long as Ulysses is located in the slow solar wind (P1, dark lines in Fig. 6). In the fast solar wind (P2, light lines) a clear periodicity is still present only in the solar wind velocity, whereas a periodicity in the magnetic field and GCR data may also be present, but is by far less obvious. These periodicities have already been discussed extensively in the literature (cf. Paizis et al., 1999; Zhang, 1997) and could be used to validate our analysis.

The picture for cycle 23 (Fig. 7) is quite different: for Ulysses flying through the slow solar wind, we can also see a periodicity, but only in the solar wind velocity and the magnetic field, and with a somewhat shorter time period of 24.5 days. The GCR variation does not show a clear periodicity, but this may be owed to the disturbing influence of transient decreases like the events (A) to (D). A period of 24.5 days may, thus, be present, too. In the fast solar wind, however, no periodicity can be seen at all.

In both cycles, we see clear periodicities, although with slightly different periods, as long as Ulysses can measure the effects of CIRs in situ, i.e. in the slow solar wind. In the fast solar wind, where in particular the GCR modulation can only be observed remotely, we see, as already suggested by Fig. 3, a different behaviour in both cycles. The result, however, is that both data sets consequently contradict the transport models, so that further analysis is necessary.

\subsection{Comparison with $1 \mathrm{AU}$ data}

The observations discussed so far raise the question of how the transport of charged particles in the inner heliosphere could take place. In order to get an idea about the large-scale processes we try to distinguish spatial from temporal variations by investigating also data measured in situ at $1 \mathrm{AU}$, keeping, however, in mind that CIRs usually only fully develop beyond the Earth orbit.

For the time interval C22 we could only make use of the Moscow Neutron monitor, because $1 \mathrm{AU}$ plasma data were measured during that period only by the IMP-8 spacecraft, which, however, stayed for significant times within the Earth's magnetosphere. Thus, CIR plasma parameters are measured with a bad coverage, making a reasonable spectral 
analysis impossible. For $\mathrm{C} 23$ we can resort to data for all three quantities studied in the previous sections: the solar wind speed and the magnetic field were measured by the instruments SWEPAM and MAG on the ACE spacecraft, while intensity of $>50 \mathrm{MeV}$ GCRs was measured by the EPHIN instruments on-board SOHO. The latter were complemented here by the count rates of the Kiel Electron Monitor.

A first impression of the data (Fig. 8) shows no significant change in the GCR modulation in cycle $\mathrm{C} 22$, whereas in C23 a transition to a more quiet phase appear to take place around 2005.7, i.e. towards the end of time period C23/P1. More profound statements, however, also require a Lomb analysis, the results of which are shown in Figs. 9 (GCRs only) and 10 for the time intervals $\mathrm{C} 22$ and $\mathrm{C} 23$, respectively. Again, dark and light colours refer to the periods $\mathrm{P} 1$ and $\mathrm{P} 2$, respectively. While Ulysses was located in P1 in the slow solar wind and in P2 in the fast one, the measurements discussed in the following were all obtained in situ at the same position, so that a comparison of the periodicities at $1 \mathrm{AU}$ with those along the Ulysses trajectory should shed light on the question whether we see temporal or spatial variations.

The data at $1 \mathrm{AU}$ behave thoroughly different from the Ulysses data. For C22, where only GCR data can be used, a clear periodicity of about 29 days (with a second one of $~ 34$ ) observed during P1 becomes weaker and shifted to about 30 days in P2, the second peak vanishes. In contrast, three clear peaks arise at 13,14, and 15 days, i.e. with about half the period as before. Such a behaviour can be expected for a northern solar coronal hole extending to the solar equator, indicating, thus, a reconfiguration of coronal structures from a one-stream to a two-stream structure.

For the time interval $\mathrm{C} 23$, a clear periodicity has been found only in the solar wind speed during P1 with a period of 27 days, corresponding to the synodic rotation of the Sun. The magnetic field data and the solar wind speed during P2 do not show a pronounced periodicity, but we see at least a low peak in the periodogram near 27 days, which comes close to the level of significance only for the solar wind speed. In addition, we see a clear periodicity of 9 days of both quantities and in both sub-periods P1 and P2. The peaks at 9 days are plotted enlarged in Fig. 11. If comparing them we see different tendencies: while the peak at 9 days is more distinct in $\mathrm{P} 1$ than in $\mathrm{P} 2$, i.e. diminishes from $\mathrm{P} 1$ to $\mathrm{P} 2$, the periodicity of 27 days appears to evolve from P1 to P2. In contrast, the GCR modulation does not show any clear periodicity at all.

Our results concerning the significance of periodicities close to that of the solar rotation (with the sidereal period for Ulysses, the synoptic one for ACE and SOHO) are summarised in Table 2. Comparing for both solar cycles the results of period P1 with that of P2 as well as the $1 \mathrm{AU}$ data with that by Ulysses, we come to the following conclusions:

- C22: At $1 \mathrm{AU}$ we see a more or less stable configuration rotating with the Sun, although some reconfiguration of
Table 2. Periodicities in the solar wind velocity $\left(v_{s w}\right)$ and magnetic field $(B)$ as well as for the detrended cosmic ray flux $(\Delta c / c)$ for the different time intervals as measured by Ulysses (upper half) and by various spacecraft (cf. text) at $1 \mathrm{AU}$ in the ecliptic plane (lower half). Plus and minus signs refer to a peak clearly above and below the line of significance in the Lomb analysis, respectively. Peaks close to this line are shown by an open circle. The brackets indicate a periodicity with a slightly ( $\gtrsim 1$ day) deviating periodicity.

\begin{tabular}{ccccc}
\hline Ulysses & $\mathrm{C} 22 / \mathrm{P} 1$ & $\mathrm{C} 22 / \mathrm{P} 2$ & $\mathrm{C} 23 / \mathrm{P} 1$ & $\mathrm{C} 23 / \mathrm{P} 2$ \\
\hline$v_{s w}$ & + & + & $(+)$ & - \\
$B$ & + & $(\circ)$ & $(+)$ & - \\
$\Delta c / c$ & + & $(\circ)$ & $(\mathrm{o})$ & - \\
\hline $1 \mathrm{AU}$ & $\mathrm{C} 22 / \mathrm{P} 1$ & $\mathrm{C} 22 / \mathrm{P} 2$ & $\mathrm{C} 23 / \mathrm{P} 1$ & $\mathrm{C} 23 / \mathrm{P} 2$ \\
\hline$v_{s w}$ & & & $(\mathrm{o})$ & $(+)$ \\
$B$ & & & - & - \\
$\Delta c / c$ & $(+)$ & $(+)$ & - & - \\
\hline
\end{tabular}

the corona seems to affect the results. As the analysis for the Ulysses data shows a quite similar behaviour, we can state that the variations we see in the data can be explained by spatial variations and that there seems to exist some kind of correlation between the regions of low and high latitudes.

- C23: In addition to a periodicity of 27 days, we also observe one with 9 days in the $1 \mathrm{AU}$ data. These two periodicities, however, evolve differently in time: while the significance of 27-day periodicity increases, the 9 day one decreases from P1 to P2. In addition, the solar wind structure changes around 2005.7, so that the variations observed at $1 \mathrm{AU}$ are temporal.

At Ulysses we see the opposite temporal evolution: the 29 day period is vanishing from $\mathrm{P} 1$ to $\mathrm{P} 2$ in $\mathrm{C} 23$. Since the temporal evolutions are not correlated with each other and the Earth is a "fixed" point with respect to latitude, we conclude Ulysses is entering a different region in space being linked at best loosely to lower latitudes. Thus, again the observations are caused by the spatial variation of the Ulysses spacecraft. The conclusion is that there is no obvious correlation between the two locations by drift, diffusion or similar effects.

\section{A possible interpretation}

The analysis in the previous section could reduce the contradiction between observations and drift-based transport models to the question of why there exists a correlation between processes taking place near the equatorial plane at $1 \mathrm{AU}$ and those at higher latitudes in cycle C22, but not in C23. Drift effects play obviously only a minor role, so that we must look for alternative explanations. 

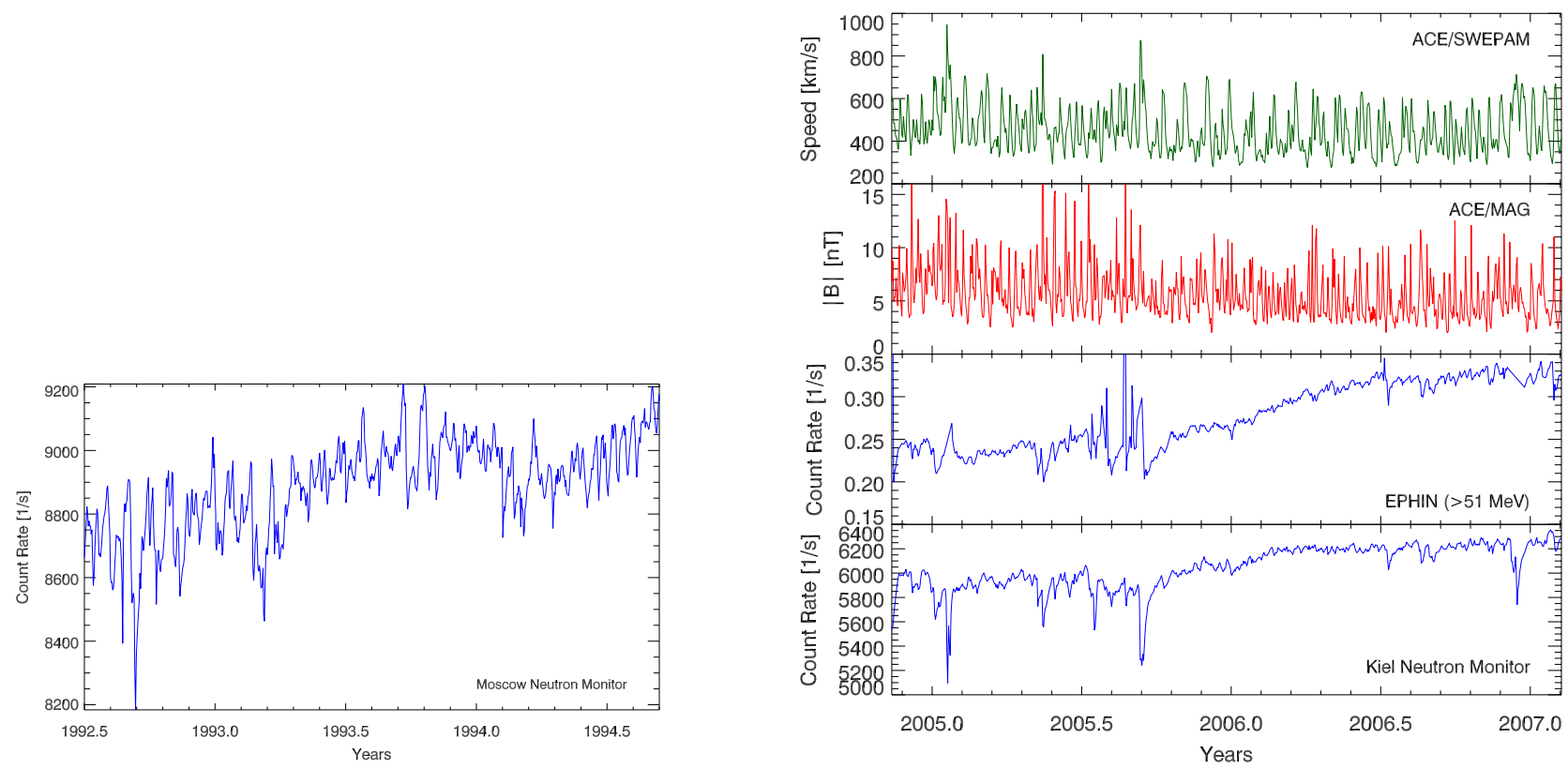

Fig. 8. The left panel the count rates of the Moscow Neutron Monitor in C22, the right panel shows the data displayed similar to Fig. 3 for the interval C23: from top to bottom the solar wind speed (SWEPAM), the magnetic field strength (MAG), the cosmic ray variation of $>51 \mathrm{MeV}$ particles (EPHAN), and the count rates of the Kiel Neutron Monitor.

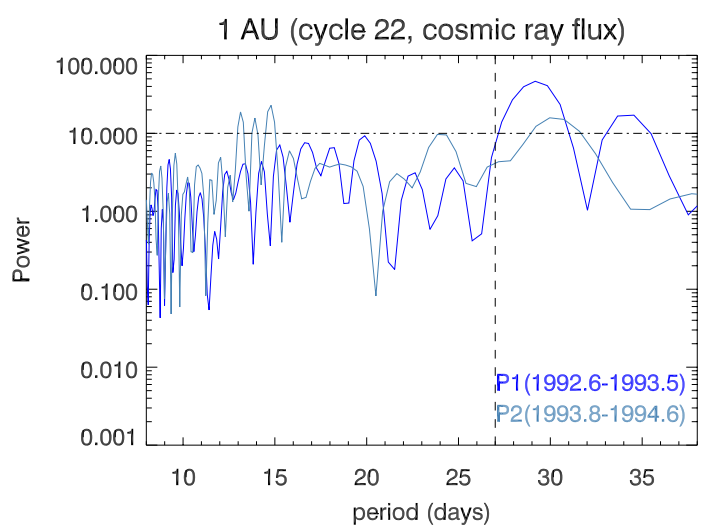

Fig. 9. Lomb periodograms for GCR measurements of the Moscow Neutron Monitor in the time interval C22. The representation is the same as in (the right panel of) Fig. 6. The vertical line indicates here the 27-day synodic period of the Sun.

The main component of the solar magnetic field beyond the source surface is, at least up to a first approximation, the radial component and further out also a longitudinal component, perpendicular to which the GCRs have to be transported into the polar direction. As drift effects are apparently not sufficiently effective, we suggest perpendicular transport to be provided by diffusion instead (cf. Jokipii and Kóta, 1995; Fisk, 1996). The question remains, however, why this transport works in $\mathrm{C} 22$, but not in $\mathrm{C} 23$.
Two facts may help to find an answer to this question: on the one hand (Jokipii and Kóta, 1995) discovered that perpendicular diffusion in the latitudinal direction is by a factor of 3-4 (Ferreira et al., 2001) more effective in the fast solar wind of a polar coronal hole than in the slow wind, provided there are stable structures much larger that the particles' gyro radius. The regions of fast solar wind are, as Helios measurements show (Schwenn, 1990), separated by sharp boundaries from those of the slow solar wind. Such boundaries occurring in the longitudinal direction generate strong gradients in the solar wind speed and finally lead to the formation of CIRs.

As CIRs are known to represent effective "barriers" for particle propagation, the idea is now to investigate such boundaries also in the latitudinal direction. The purpose is again twofold: Are there actually large and stable regions of fast solar wind, i.e. coronal holes, where effective perpendicular diffusion can take place? And: do we possibly see boundaries, which can be regarded as "barriers" for the latitudinal particle transport?

These questions are addressed by investigating the coronal hole evolution deduced from Carrington maps of the YOHKOH Soft X-ray Telescope (SXT) in C22 and the SOHO Extreme ultraviolet Imaging Telescope (EIT) in C23. The left panel of Fig. 12 shows the SXT maps for Carrington rotations 1868 (April/May 1993), 1874 (September/October 1993), and 1880 (March 1994), whereas the right panel shows EIT maps. Displayed are the Carrington rotations 


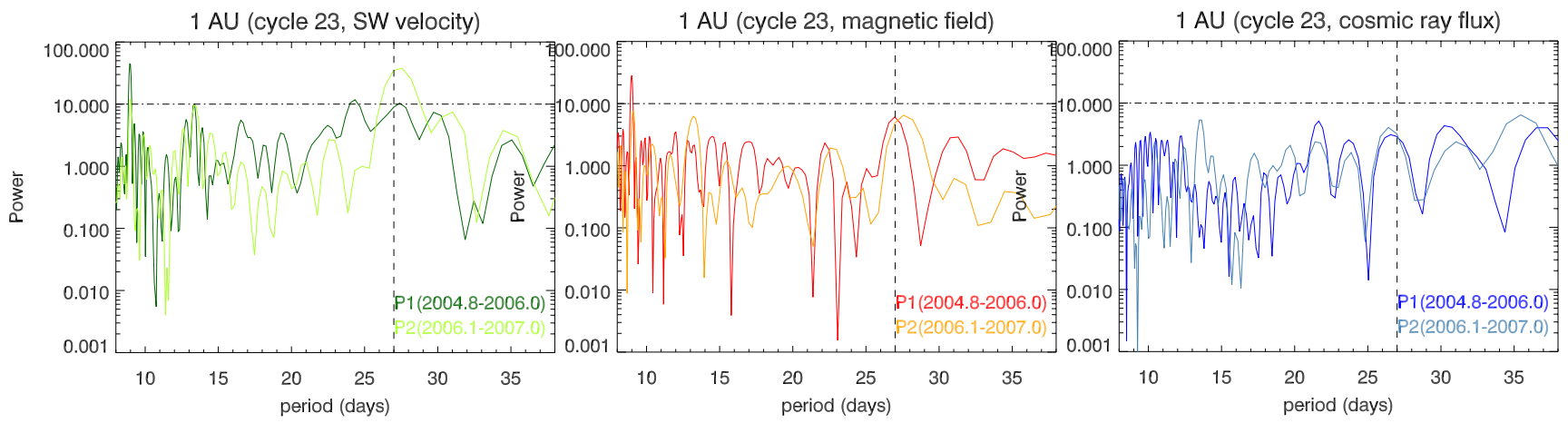

Fig. 10. Lomb periodograms for the solar wind speed (SWEPAM) and magnetic field (MAG), measured on the ACE spacecraft as well as the GCR flux (EPHIN instrument on SOHO), the representation is the same as in Fig. 6, the vertical line shows again 27 days.

2025 (January 2005), 2039 (January/February 2006), and 2045 (July 2006). In both solar cycles, the upper and lower maps represent the periods $\mathrm{P} 1$ and $\mathrm{P} 2$ respectively, while both maps in the middle show the transition between them.

The maps for C22 (Yohkoh SXT) show an extended coronal hole structure reaching from southern polar regions to the equator. Although this coronal hole moves slowly, i.e. within one year, from about $45^{\circ}$ in CR 1868 to about $0^{\circ}$ in CR 1880 , its form remains almost the same, so that we see an extended, stable structure, within which effective perpendicular diffusion can take place. For C23 (SOHO EIT) we see, in contrast, only small and variable equatorial coronal holes, which do not extend to higher latitudes. Therefore, we conclude tentatively that the modulation of GCRs is correlated with the spatial extensions of these holes. In C23 the holes are large enough to allow the acceleration of low-energy particles, but too small for an efficient acceleration of high-energy particles (Lario and Roelof, 2007) or modulation of GCRs.

The Carrington maps, thus, suggest the following interpretation: during $\mathrm{C} 22$ a large southern coronal hole extending up to equatorial regions allows the transport of charged particles in the latitudinal direction by perpendicular diffusion, while in $\mathrm{C} 23$ no such structure is present. Instead, small hole structures with in part sharp boundaries do not permit effective perpendicular diffusion as was present in C22. Thus, we may conclude that drift effects are in both solar cycles of minor importance.

\section{Summary and conclusion}

Decreases in the intensity of galactic cosmic rays can be divided into two groups: while transient decreases are caused by more or less isolated events like interplanetary coronal mass ejections, recurrent decreases are caused by corotating interaction regions (CIRs). The periodic nature of the latter make recurrent decreases a useful tool for studying the transport of charged particles in the heliosphere.

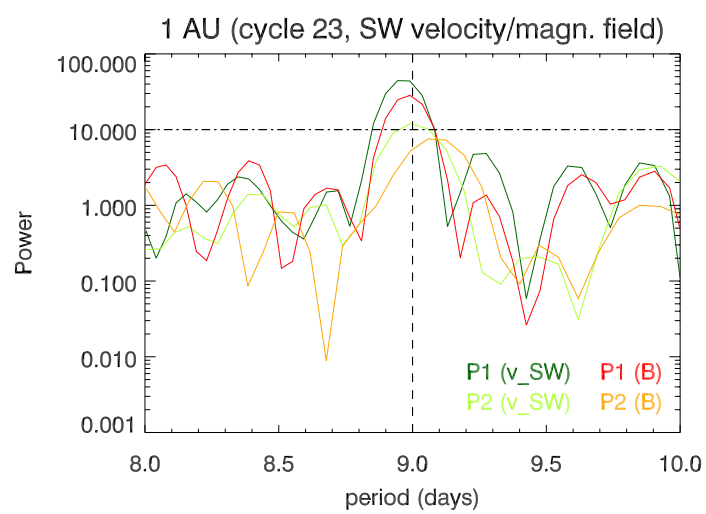

Fig. 11. Lomb periodograms the solar wind speed (green) and the magnetic field (red) in C23, detail of Fig. 10 around a periodicity of 9 days.

Of particular interest is the transport in the latitudinal direction, i.e. from the equatorial plane to the polar regions of the heliosphere and vice versa: while the corotating interaction regions occur usually only at low latitudes, observations at higher latitudes can reflect the CIR modulation only remotely and provide, thus, valuable informations about the latitudinal transport of galactic cosmic rays, i.e. perpendicular to the heliospheric magnetic field.

The inclined trajectory of the Ulysses spacecraft provided for the first time the opportunity not only to measure the cosmic ray intensity, but also the solar wind speed and the magnetic field. The perpendicular transport of charged particles should essentially be provided by diffusion and, in particular, by drift effects. Analytical as well as numerical models have been developed in order to model the drift motion in the heliosphere, which depends on the sign of the solar magnetic field.

The data obtained during the first descent of the Ulysses spacecraft in the $A>0$ solar cycle 22 , however, did not show the expected result: the periodicity in the cosmic ray intensity 

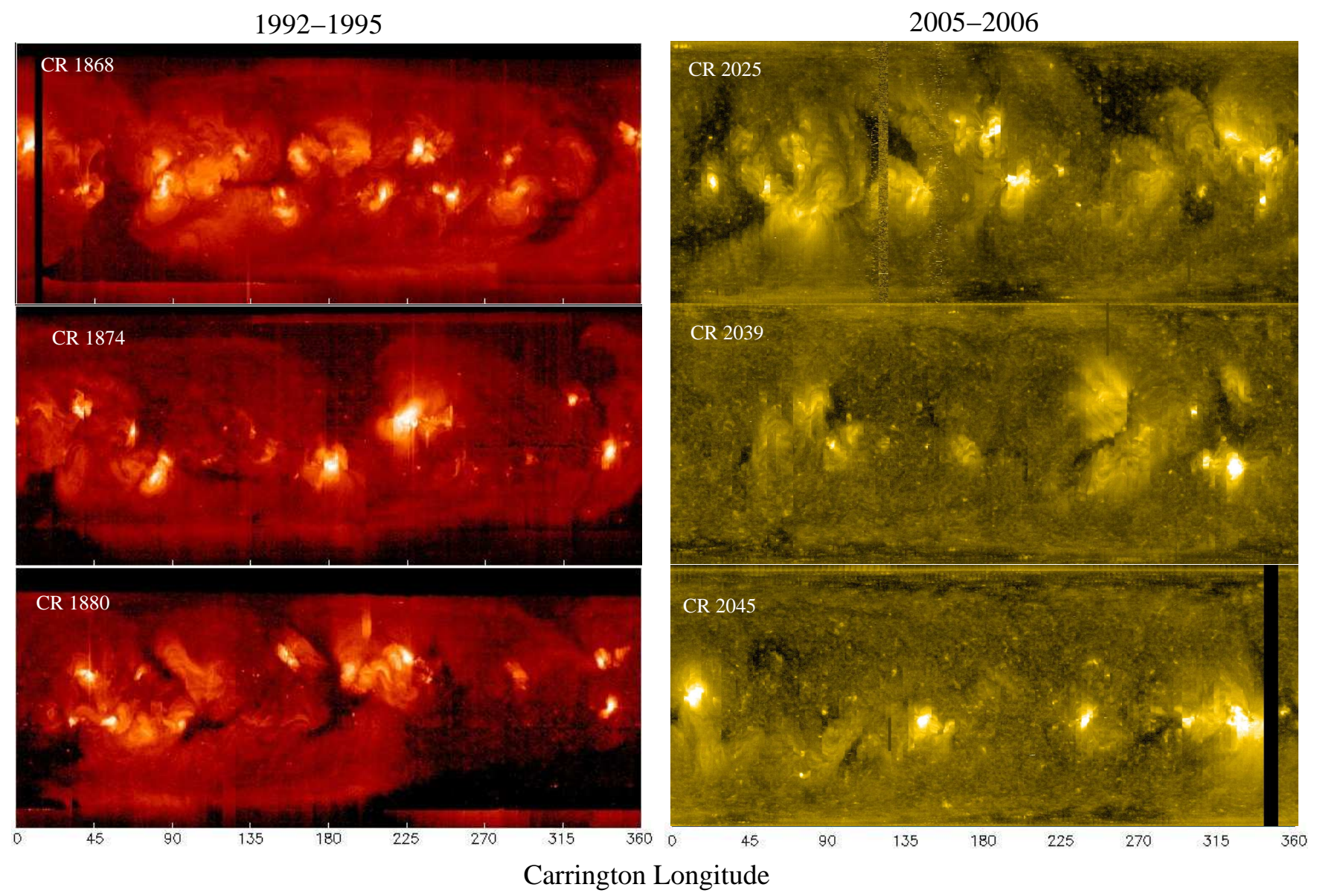

Fig. 12. Left panel: Yohkoh SXT Carrington maps for rotations number 1868, 1874, and 1880, standing for the periods C22/P1, the transition and C22/P2. Right panel: SOHO EIT Carrington maps at $284 \AA$ for rotations number 2025, 2039, and 2045, resprenting the time intervals $\mathrm{C} 23 / \mathrm{P} 1$, the transition and $\mathrm{C} 23 / \mathrm{P} 2$.

observed in the slow solar wind (period P1) was still present at high latitudes, i.e. in the fast solar wind (period P2). The second Ulysses flyby about 12 years later offered the opportunity to repeat the measurements along the same trajectory and in similar solar wind conditions, but with reversed solar magnetic field polarity during the $A<0$ solar cycle 23 .

These second observations, however, were as surprising as the first ones were: the periodicity observed in P1 almost vanishes in P2, a result that was expected, however, for the opposite polarity of the solar magnetic field, i.e. during cycle 22. In order to get an idea how this apparently opposing behaviour in both solar cycles arises, we performed a detailed analysis of the data by investigating (1) the amplitudes and (2) the periodicities of the cosmic ray decreases and comparing (3) the Ulysses data with those obtained along the Earth orbit in order to distinguish spatial and temporal variations. The results can be summarised as follows:

- During solar cycle 23 the amplitudes of the cosmic ray decreases were lower than in cycle 22 , indicating an increased solar activity. The dominating criterion for
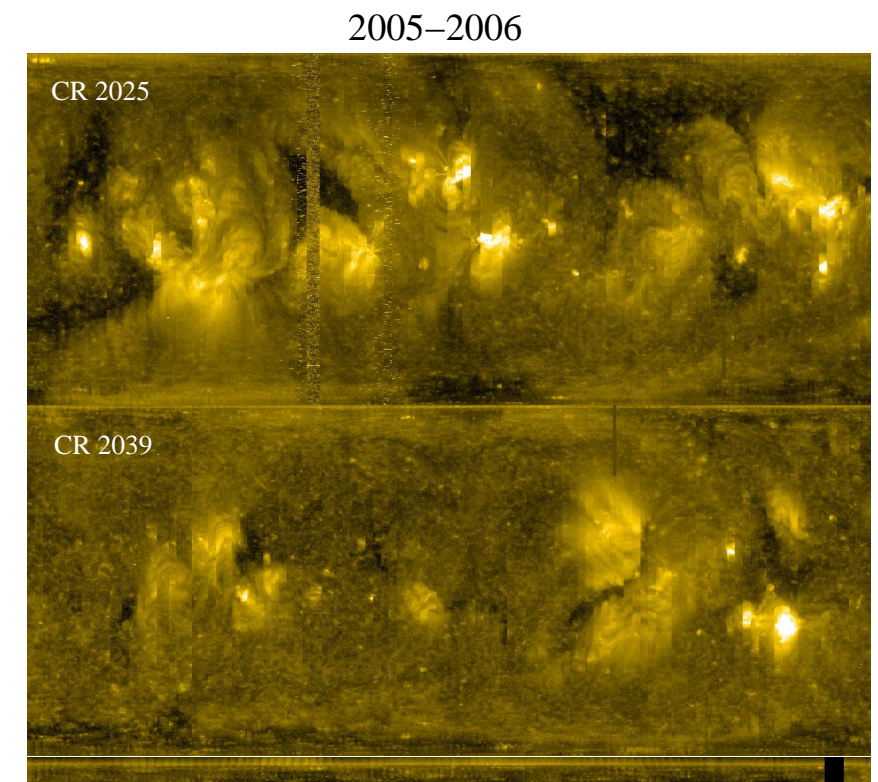
.

.


however, we see temporal variations at $1 \mathrm{AU}$, but spatial variations along the Ulysses trajectory, i.e. neither a stable configuration nor a correlation between the slow and fast solar wind regions.

Drift-dominated particle-transport models obviously fail, on the one hand, to explain our measurements, but on the other hand, the large-scale stable structures seen in cycle 22 suggest latitudinal diffusion to take place instead. This process can work much more efficient in extended regions within the fast solar wind, so that we investigated in addition the coronal hole structures for the respective time intervals by using Carrington maps by Yohkoh SXT (cycle 22) and SOHO EIT (cycle 23). The maps actually show a large and (almost) stable coronal hole extending from the south pole into equatorial regions in cycle 22 , but only small-scale structures with boundaries, which can be regarded as "barriers" for the particle transport in cycle 23.

As a possible explanation, which certainly must be critically inspected by further studies, we suggest that the modulation processes are almost the same, so that different coronal hole structures leading to different CIR structures rather than drift effects are the reason for the opposing behaviour in both cycles. This conclusion is supported by Lario and Roelof (2007) showing that CIRs in cycle 23 were, in contrast to cycle 22 , only able to accelerate low energy particles. While a large and stable coronal hole allows an effective latitudinal transport in cycle 22, small-scale structures and boundaries allow almost no correlation between slow and fast solar wind regions in cycle 23 .

Acknowledgements. The ULYSSES/KET project is supported under grant No. 50 OC 0105 by the German Bundesminsterium für Wirtschaft through the Deutsches Zentrum für Luft und Raumfahrt (DLR). It is our pleasure to thank the Ulysses Data System team for providing valuable data. We thank the ACE SWEPAM instrument team and the ACE Science Center for providing the ACE data. Moreover, we would like to thank Oliver Sternal for his assistance with the preparation of Fig. 1.

Topical Editor R. Forsyth thanks two anonymous referees for their help in evaluating this paper.

\section{References}

Alania, M. V., Gil, A., Iskra, K., and Modzelewska, R.: 27Day Variations Of The Galactic Cosmic Ray Intensity And Anisotropy In Different Solar Magnetic Cycles (1964-2004), in: International Cosmic Ray Conference, vol. 2 of International Cosmic Ray Conference, pp. 215-218, 2005.

Balogh, A., Beek, T. J., Forsyth, R. J., Hedgecock, P. C., Marquedant, R. J., Smith, E. J., Southwood, D. J., and Tsurutani, B. T.: The magnetic field investigation on the ULYSSES mission - Instrumentation and preliminary scientific results, Astron. Astrophys. Suppl., 92, 221-236, 1992.

Bame, S. J., McComas, D. J., Barraclough, B. L., Phillips, J. L., Sofaly, K. J., Chavez, J. C., Goldstein, B. E., and Sakurai, R. K.:
The ULYSSES solar wind plasma experiment, Astron. Astrophys. Suppl., 92, 237-265, 1992.

Clem, J. M. and Dorman, L. I.: Neutron Monitor Response Functions, Space Sci. Rev., 93, 335-359, doi:10.1023/A: 1026508915269, 2000.

Ferreira, S. E. S., Potgieter, M. S., Burger, R. A., Heber, B., and Fichtner, H.: Modulation of Jovian and galactic electrons in the heliosphere: 1. Latitudinal transport of a few $\mathrm{MeV}$ electrons, J. Geophys. Res., 106, 24979-24 988, doi:10.1029/ 2001JA000082, 2001.

Fisk, L. A.: Motions of the footpoints of heliospheric magnetic field lines at the Sun: Implications for recurrent energetic particle events at high heliographic latitudes, J. Geophys. Res., 101(A7), 15 547-15 553, 1996.

Fisk, L. A. and Jokipii, J. R.: Mechanisms for latitudinal transport of energetic particles in the heliosphere, Space Sci. Rev., 89, 115-124, 1999.

Forbush, S. E.: On the Effects in Cosmic-Ray Intensity Observed During the Recent Magnetic Storm, Phys. Rev., 51, 1108-1109, 1937.

Gil, A., Iskra, K., Modzelewska, R., and Alania, M. V.: On the 27day variations of the galactic cosmic ray anisotropy and intensity for different periods of solar magnetic cycle, Adv. Space Res., 35, 687-690, doi:10.1016/j.asr.2005.03.018, 2005.

Heber, B., Sanderson, T. R., and Zhang, M.: Corotating Interaction Regions, Adv. Space Res., 23, 567-579, 1999.

Jokipii, J. R. and Kóta, J.: Three-dimensional cosmic-ray simulations: heliographic latitude and current-sheet tilt, Space Sci. Rev., 72, 379-384, 1995.

Jokipii, J. R., Levy, E. H., and Hubbard, W. B.: Effects of particle drift on cosmic ray transport, I. General properties, application to solar modulation, Astrophys. J., 213, 861-868, 1977.

Kunow, H., Dröge, W., Heber, B., Müller-Mellin, R., Röhrs, K., Sierks, H., Wibberenz, G., Ducros, R., Ferrando, P., Rastoin, C., Raviart, A., and Paizis, C.: High energy cosmic ray nuclei results on ULYSSES: 2. Effects of a recurrent high-speed stream from the southern polar coronal hole, Space Sci. Rev., 72, 397-402, 1995.

Lanzerotti, L. J., Gold, R. E., Anderson, K. A., Armstrong, T. P., Lin, R. P., Krimigis, S. M., Pick, M., Roelof, E. C., Sarris, E. T., and Simnett, G. M.: Heliosphere Instrument for Spectra, Composition and Anisotropy at Low Energies, Astron. Astrophys. Suppl., 92, 349-363, 1992.

Lario, D. and Roelof, E. C.: Energetic particles during the first and third Ulysses southern high-latitude excursions: Probing global corotating interaction region structure beyond 5 AU, J. Geophys. Res., 112(A11), A09107, doi:10.1029/2007JA012414, 2007.

Le Roux, J. A. and Potgieter, M. S.: The simulation of Forbush decreases with time-dependent cosmic-ray modulation models of varying complexity, Astron. Astrophys., 243, 531-545, 1991.

Lomb, N. R.: Least-squares frequency analysis of unequally spaced data, Astrophys. Space Sci., 39, 447-462, 1976.

Malandraki, O. E., Marsden, R. G., Tranquille, C., Forsyth, R. J., Elliott, H. A., Lanzerotti, L. J., and Geranios, A.: Energetic particle observations by Ulysses during the declining phase of solar cycle 23, J. Geophys. Res., 112, A06111, doi:10.1029/ 2006JA011876, 2007.

McComas, D. J., Bame, S. J., Barker, P., Feldman, W. C., Phillips, J. L., Riley, P., and Griffee, J. W.: Solar Wind Electron Pro- 
ton Alpha Monitor (SWEPAM) for the Advanced Composition Explorer, Space Sci. Rev., 86, 563-612, doi:10.1023/A: 1005040232597, 1998.

Müller-Mellin, R., Kunow, H., Fleissner, V., Pehlke, E., Rode, E., Roschmann, N., Scharmberg, C., Sierks, H., Rusznyak, P., McKenna-Lawlor, S., Elendt, I., Sequeiros, J., Meziat, D., Sanchez, S., Medina, J., del Peral, L., Witte, M., Marsden, R., and Henrion, J.: COSTEP - Comprehensive Suprathermal and Energetic Particle Analyser, Solar Phys., 162, 483-504, 1995.

Paizis, C., Heber, B., Ferrando, P., Raviart, A., Falconi, B., Marzolla, S., Potgieter, M. S., Bothmer, V., Kunow, H., MüllerMellin, R., and Posner, A.: Amplitude evolution and rigidity dependence of the 26-day recurrent cosmic ray decreases: COSPIN/KET results., J. Geophys. Res., 104, 28 241-28247, 1999.

Phillips, J. L., Bame, S. J., Barnes, A., Barraclough, B. L., Feldman, W. C., Goldstein, B. E., Gosling, J. T., Hoogeveen, G. W., McComas, D. J., Neugebauer, M., and Suess, S. T.: Ulysses solar wind plasma observations from pole to pole, Geophys. Res. Lett., 22, 3301-3304, 1995.

Potgieter, M. S. and Moraal, H.: A drift model for the modulation of galactic cosmic rays, Astrophys. J., 294, 425-440, 1985.

Richardson, I. G.: Energetic Particles and Corotating Interaction Regions in the Solar Wind, Space Sci. Rev., 111, 267-376, doi: 10.1023/B:SPAC.0000032689.52830.3e, 2004.

Richardson, I. G., Cane, H. V., and Wibberenz, G.: A 22-year dependence in the size of near-ecliptic corotating cosmic ray depressions during five solar minima, J. Geophys. Res., 104, 12 549-12 562, doi:10.1029/1999JA900130, 1999.
Schwenn, R.: Large scale structure of the interplanetary medium, in: Physics of the inner heliosphere, edited by Schwenn, R. and Marsch, E., chap. 3, pp. 99-183, Springer, Berlin, 1990.

Simpson, J. A.: Cosmic-Radiation Intensity-Time Variations and Their Origin. III. The Origin of 27-Day Variations, Phys. Rev., 94, 426-440, 1954.

Simpson, J. A., Anglin, J., Balogh, A., Bercovitch, M., Bouman, J. M., Budzinski, E. E., Burrows, J. R., Carvell, R., Connell, J. J., Ducros, R., Ferrando, P., Firth, J., Garcia-Munoz, M., Henrion, J., Hynds, R. J., Iwers, B., Jacquet, R. M., Kunow, H., Lentz, G. A., Marsden, R. G., McKibben, R. B., Müller-Mellin, R., Page, D. E., Perkins, M. A., Raviart, A., Sanderson, T. R., Sierks, H., Treguer, L., Tuzzolino, A. J., Wenzel, K.-P., and Wibberenz, G.: The Ulysses Cosmic-Ray and Solar Particle Investigation, Astron. Astrophys. Suppl., 92, 365-399, 1992.

Smith, C. W., L'Heureux, J., Ness, N. F., Acuña, M. H., Burlaga, L. F., and Scheifele, J.: The ACE Magnetic Fields Experiment, Space Sci. Rev., 86, 613-632, doi:10.1023/A:1005092216668, 1998.

Stone, E. C., Frandsen, A. M., Mewaldt, R. A., Christian, E. R., Margolies, D., Ormes, J. F., and Snow, F.: The Advanced Composition Explorer, Space Sci. Rev., 86, 1-22, doi:10.1023/A: $1005082526237,1998$.

Struminsky, A. B.: Intensity enhancements of 200-2000 MeV protons in 1997-2005 as measured by the KET/ULYSSES, Cosmic Res., 45, 368-372, doi:10.1134/S0010952507040119, 2007.

Zhang, M.: A Linear Relationship between the Latitude Gradient and 26 Day Recurrent Variation in the Fluxes of Galactic Cosmic Rays and Anomalous Nuclear Components. I. Observations, Astrophys. J., 488, 841-853, doi:10.1086/304732, 1997. 